\title{
Long-term follow-up of a low profile, coated, press-fit cup: the trabeculae oriented pattern (T.O.P.) acetabular system
}

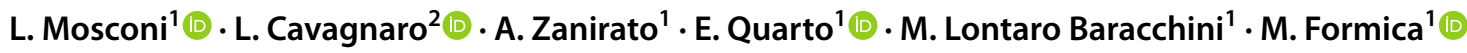

Received: 4 December 2020 / Accepted: 12 February 2021 / Published online: 3 March 2021

(c) The Author(s) 2021

\begin{abstract}
Purpose Total hip arthroplasties (THAs) are rising worldwide, as the functional request of patients who undergo this procedure. The trabeculae oriented pattern (TOP) is a modern cup, which follows the philosophy of the tissue sparing surgery (TSS). Focusing on clinical and radiological results and complications, the authors aim to highlight the outcomes of the TOP at a long-term follow-up (FU).

Methods A retrospective analysis was completed on THA performed with the TOP cup between 1997 and 2015. Five hundred and eighty-eight patients sustained surgery, for a total of 662 cup implanted. Four hundred and sixty patients (524 hips) were examined. Mean FU was $12 \pm 4.9$ years (range 5-22). Clinical (HHS, OHS and VAS) and radiological data were obtained. Every complication, reoperation or revision was recorded and analyzed.

Results Clinical evaluation revealed a HHS of 87.1 \pm 13.8 an OHS of $41.3 \pm 5.4$, and a VAS of $1.2 \pm 1.1$. Acetabular osteolysis was observed in 53 hips. Overall survival rate of the cup was $90.5 \%$ (50 revisions), the main causes of cup substitution being aseptic loosening (AL) of the cup combined with the stem (26), of the cup only (13 cases) and periprosthetic joint infection (7 cases).

Conclusion TOP cup has demonstrated a good overall survivorship at a long-term FU, even compared with other coated cups, providing excellent clinical result with low rate of complications. Its association with a neck sparing stem permits a physiologic load transmission, reducing the stress shielding effect that could cause early implant mobilization.
\end{abstract}

Keywords Total hip arthroplasty $\cdot$ Tissue sparing surgery $\cdot$ Trabeculae oriented pattern · Coated acetabular cup

\section{Introduction}

Total hip arthroplasty (THA) has been described as the "operation of the century," because of its excellent results in improving patients' quality of life [1]: nowadays, uncemented components are the preferred choice for hip substitution, especially in younger patients.

Surgeons therefore must often measure themselves with patients' high functional requests and expectations for a rapid recovery and return to preoperative activity levels. As well as stem components, also acetabular cups design evolved. Currently, the majority of acetabular cups relies on

L. Mosconi

lorenzomosconi11@gmail.com

1 Clinica Ortopedica E Traumatologica, Ospedale Policlinico San Martino, Genova, Italy

2 Ospedale Santa Corona, Pietra Ligure (SV), Italy a press-fit mechanism for primary stability, with the possibility of adding cancellous screws. The bone-implant interface is made by osteoconductive or osteoinductive materials, such as porous or hydroxyapatite (HA) coating, porous tantalum or titanium. The trabeculae oriented pattern (TOP, Waldemar Link, Hamburg, Germany) cup was created following the philosophy of tissue sparing surgery (TSS) [2] and the idea that the prosthesis should reproduce native hip load transmission [3]. The concept of TSS has been introduced with the idea of substitute only pathologic tissue, in order to spare reliable bone facing the possibility of further revisions [2].

At our Institution, the first TOP cup, in combination with the collum femoris preserving (CFP, Waldemar Link, Hamburg, Germany) stem, was implanted in the late $90 \mathrm{~s}$ : the purpose of this paper is to report the long-term clinical and radiological outcomes as well as complications of TOP cup. 


\section{Materials and methods}

\section{Study design}

A retrospective analysis was performed on a cohort of patients who underwent THA at our institution between 1998 and 2015. All patients provided their written and informed consent preoperatively to every medical and surgical procedures.

Inclusion criteria for patients' selection were: THA with TOP cup, minimum follow-up (FU) of 5 years, complete clinical and radiological data. Patients with severe hip dysplasia (Crowe III or IV) or other severe joint deformities were excluded from case series. Demographic, clinical and surgical data were collected, including age at surgery and at final FU, sex, relevant comorbidities, smoking status, body mass index (BMI), preoperative diagnosis, surgical time, stem, liner, head features and cup dimension of all patients.

\section{TOP surgical philosophy}

The TOP is a hemispherical cup made of Tilastan, a Ti $6 \mathrm{Al} 4 \mathrm{Va}$ alloy with a $160 \mu \mathrm{m}$ porous surface coated with a $15-\mu \mathrm{m}$-thick calcium phosphate layer. It has a medial ventral recess, which diminishes the possibility of impingement between the cup and the collar stem, the iliopsoas tendon or the femoral nerve, tree teeth on the equator (similarly to those of treated cups) and tree holes for fixation screws, in order to improve primary stability. The liner is in ultra high molecular weight polyethylene (UHMWPE) or X-linked UHMWPE and presents a dissociation of $20^{\circ}$ with the equator of the shell: the rationale is to improve the range of motion and permits the implantation of the cup even in dysplastic acetabulum, in which the inclination is often superior to the $55^{\circ}$ recommended for shell positioning. The TOP presents 15 different sizes (from 40 to $68 \mathrm{~mm}$ of diameter, with a $2 \mathrm{~mm}$ increment): the inner diameter of the insert allows the use of ceramic or metal heads from 22 to $36 \mathrm{~mm}$. A line-to-line reaming is recommended for correct cup implantation: trial cups and liners are available for intraoperative check.

\section{Surgical procedure}

An accurate preoperative planning with component templates was developed, collegially debated, and a rescue option was prepared for every implant. Every surgery was performed through a modified Hardinge lateral approach, with the goal of minimizing muscle damage [4]. The aim of this approach is to minimize soft tissue damage and remove only pathologic tissue, in order to increase recovery and rehabilitation. A short-term antibiotic (ATB) prophylaxis was administered. Patients were mobilized with partial weight bearing using two crutches for the first week, following a rapid recovery of full ambulation: antithrombotic prophylaxis was carried out with low molecular weight heparin (LMWH) and compression socks for 45 days after the surgery.

\section{Clinical and radiological evaluation}

Clinical evaluation was accomplished using the Harris Hip Score (HHS) to assess joint function from the clinician point of view, the Oxford Hip Score (OHS), patient-centered outcome, the visual analog scale (VAS) for assessment of groin or thigh pain [5]. HHS results were classified as excellent (HHS $\geq 90)$, good (89-80), fair (79-70) and poor $(<70)$, and OHS in satisfactory (40-48), mild (30-39), fair (20-29) and poor (0-19).

Every patient radiological evaluation was conducted on an anteroposterior (AP) plain pelvis X-ray with the legs in a slight $\left(15^{\circ}\right)$ internal rotation and a modified Dunn' view [6]. The radiographs were analyzed by two experienced surgeons (MF and LF), blinded for clinical outcomes, for cup inclination (intended as the angle formed between the acetabulum and the transischial line [6]) and loosening, osteolysis according to DeLee and Charnley [7], Gruen' zones for the stem [8], heterotopic ossifications (HO) according to Brooker classification [9], neck reabsorption ratio (NRR, only evaluated in case of neck sparing stems) [10] and leg length discrepancy (LDD) [11].

Every prosthesis-related complication, such as septic or aseptic loosening, dislocation, intra-/postoperative fractures, was recorded, as well as any reoperation (intended as any further surgery on the operated hip) or revision (any surgery that required fixed component exchange).

\section{Statistical analysis}

Cup survival was reported as a Kaplan-Meier curve using GraphPad Prism 8, with cup revision as an end point. Continuous variables were expressed as mean \pm standard deviation (SD), while categorical variables were reported as percentages or frequencies, using Microsoft Excel for Office 2019. For radiological parameters, Cohen's kappa coefficient was used to assess correlation between the two testers, and it demonstrates a correlation of more than $90 \%$.

\section{Results}

Five hundred and eighty-eight patients underwent THA with the TOP cup between 1997 and 2014 at our institution: among them, $514(87.4 \%)$ underwent unilateral hip 
substitution and 74 (12.6\%) bilateral. Two hundred and eighty-nine (49.1\%) were males and 299 (50.9\%) females. Globally, 662 TOP cups were implanted during the examined period (374 on the right hip, 288 on the left one). Surgical diagnosis causing replacement was primary osteoarthritis (OA) in 541 patients $(81.3 \%)$, femoral neck fracture in 60 (9.1\%), femoral head necrosis in $33(4.9 \%)$, post-traumatic OA in $14(2.1 \%)$ and dysplasia in $13(1.9 \%)$ epiphysiolysis in $1(0.1 \%)$. Mean FU was $12 \pm 4.9$ years. At the last follow-up, 85 (14.4\%) of patients were dead and $43(7,3 \%)$ were lost, resulting in a total drop-out rate of $21,7 \%$ (128 patients), leaving 460 patients(524 hips) available for final statistical analysis. Patients sustained surgery at a mean age of $66 \pm 15.5$ years, and the mean age at the last FU was $75 \pm 10.7$ years. Regarding smoke status, $337(73.3 \%)$ were non-smokers, 110 smokers $(23.8 \%)$ and 13 (2.9\%) former smokers: mean BMI was $26.5 \pm 3.7$. Mean surgical time was $89.1 \pm 35.3 \mathrm{~min}$. Cup size, liner and head features are resumed in Table 1; stem pairing in Table 2. Relevant comorbidities are summarized in Table 3 .

\section{Clinical and radiographic results}

HHS at the end of FU was $87.1 \pm 13.8$, VAS $1.2 \pm 1.1$, OHS $41.3 \pm 5.4$. HHS results proved excellent in 237 subjects (51.6\%), good in 160 (34.7\%), fair in $26(5.6 \%)$ and poor in $37(8.1 \%)$ : OHS outcomes were satisfactory for 307 patients $(66.7 \%)$, mild in $126(27.5 \%)$, fair in $21(4.5 \%)$ and poor in

Table 1 Cup dimension, liner and head features
Table 2 Stem pairing

\begin{tabular}{lrr}
\hline Stem & \multicolumn{1}{c}{$N$} & \multicolumn{1}{c}{$\%$} \\
\hline CFP & 430 & 64.9 \\
CBC & 49 & 7.4 \\
CLS & 23 & 3.5 \\
LC & 15 & 2.3 \\
SP2 & 118 & 17.8 \\
SPS & 20 & 3.1 \\
Stellaris & 6 & 0.9 \\
Wagner & 1 & 0.1 \\
\hline
\end{tabular}

(CFP and SP2, Waldemar Link, Hamburg, Germany; CBC and Stellaris, Mathys ltd, Bettlach, Switzerland; CLS and Wagner, Zimmer Inc., Warsaw, IN, USA; SPS, Symbios Orthopaedics, Exeter, UK; LC, Samo SPA, Bologna, Italy)

$6(1.3 \%)$. In 14 cases (3.6\%), the patients reported persistent thigh pain. No cases of psoas or femoral nerve impingement were observed.

Osteolysis on the acetabular side was observed in 53 (10.1\%) hips, concerning zone I in 26 cases $(4.9 \%)$, zone II in 94 (17.9\%), 19 in zone III (3.6\%): in some cases, it involved more than one area in the same hip. Mean acetabular inclination was $46.5^{\circ} \pm 4.3^{\circ}$. On the femur, osteolysis was more frequent in Gruen zone I (34.9\% of the cases), zone II (17.7\%) and zone VII (23.6\%). Mean LDD was $1,45 \mathrm{~mm}$, with a SD of $1.93 \mathrm{~mm}$, and NRR, evaluated in the sub-cohort of patients treated with neck sparing stem, $0.26 \pm 0.28$. HO were present in $176(33.6 \%)$ cases, more frequently classifiable as Brooker I (70 hips) or II (67).

\section{Complications}

Sixty-six (12.5\%) patients underwent revision surgery for any reason. Among them 50 had developed aseptic loosening (AL) of the cup $(13,2.4 \%)$, of the stem $(11,2.1 \%)$ or both $(26,4.9 \%)$. Periprosthetic joint infection occurred in 8 patients (1.6\%), and $7(1.3 \%)$ cases were treated with a twostage revision procedure: in one patient, a suppressive therapy was preferred due to critical clinical conditions. Every case of PJI was confirmed with a preoperative arthrocentesis.

Other causes of cup revision include polyethylene wear ( 2 cases, $0.4 \%)$ and recurrent dislocation $(2,0.4 \%)$. Polyethylene wear occurred in 17 patients globally, but in 15 of them, liner substitution was sufficient to restore a good hip function: 2 cases of recurrent dislocation were treated conservatively. Complications are resumed in Table 4.

Acetabular bone loss was classified according to Paprosky classification [12]. In every case, cup revision 
Table 3 Smoking status and relevant comorbidities

\begin{tabular}{lrr}
\hline Smoking status & $N$ & $\%$ \\
\hline Current & 110 & 23.8 \\
Former & 13 & 2.9 \\
\hline Comorbidities & $N$ & $\%$ \\
\hline Diabetes & 43 & 6.5 \\
Autoimmune diseases & 32 & 4.8 \\
Chronic kidney disease & 24 & 3.6 \\
Hepatopathy & 11 & 1.7 \\
Sickle cell disease & 8 & 1.2 \\
Mild cerebral palsy & 2 & 0.3 \\
\hline
\end{tabular}

Table 4 Complications

\begin{tabular}{lrr}
\hline & $N$ & $\%$ \\
\hline Complications & 66 & 12.5 \\
Revisions & 13 & 2.4 \\
Aseptic loosening cup & 11 & 2.1 \\
Aseptic loosening stem & 26 & 4.9 \\
Aseptic loosening cup + stem & 7 & 1.3 \\
Periprosthetic joint infection & 2 & 0.4 \\
Polyethylene wear & 2 & 0.4 \\
Recurrent dislocation & 5 & 0.9 \\
Periprosthetic fracture & & \\
Reoperations & 1 & 0.2 \\
Ceramic rupture & 15 & 2.9 \\
Polyethylene wear (liner substitution) & 2 & 0.4 \\
Recurrent dislocation (conservative) & 1 & 0.2 \\
Periprosthetic joint infection (suppressive) & & \\
\hline
\end{tabular}

was performed using a Trilogy Trabecular Metal cup (Zimmer Inc., Warsaw, IN, USA).

Globally, in our cohort, there was a cup revision rate of $9.5 \%$ (50 cases), with a $90.5 \%$ survival rate at the end of FU. Mean cup revision time was $10.1 \pm 4.3$ years. Figures 1 and 2 shows the Kaplan-Meier curve indicating cup survival.

Other reasons for revision surgery were stem loosening (11 patients) and periprosthetic fracture (5).

One patient suffered of ceramic head rupture and required one further surgical procedure.

There was no correlation $(p=0.09)$ between complication insurgence and patients' comorbidities: a significant correlation $(p=0.001)$ was found between HHS results and osteolysis, demonstrating a tight correlation between clinical and radiological results.

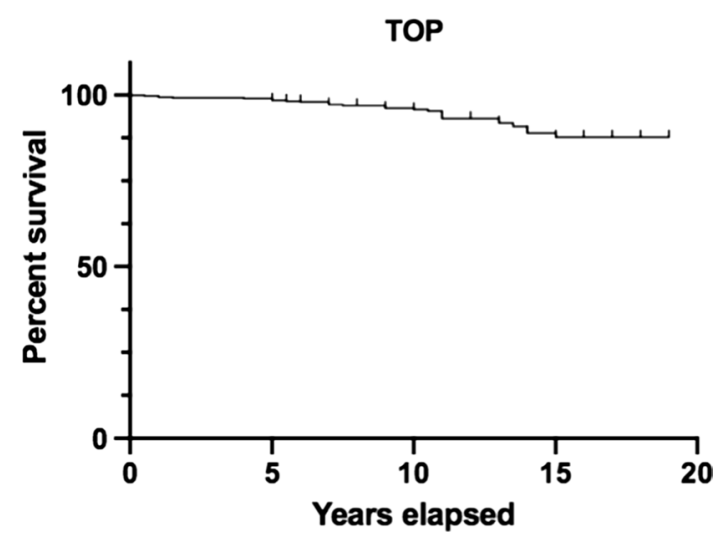

Fig. 1 Kaplan-Meier curve of TOP cup survival

\section{Discussion}

TOP cup showed a good survivorship at a long-term FU $(90.5 \%)$. As far as we know, this is one of the few reports on a long-term FU [range 5-21 years] for this type of cup. The use of TOP cup has been previously validated in short-term clinical studies [13-16], and with analysis performed on bone reabsorption [17]. Some authors advocated an increased risk of revision in cups coated with hydroxyapatite $[18,19]$. The difference we report in terms of cup survival could be related to the fact that the TOP cup relies on a different principle than the cups examined by Lazarinis and coll. (partially treated, slight HA coating, partial hemispheric profile). As highlighted by the authors, in the population they examined differences were seen among the various types of cups examined: furthermore, the use of femoral heads of smaller sizes and different tribology (metal heads) could make the two cohorts of patients dissimilar. Main reason for cup revision was $\mathrm{AL}$ (isolated or combined with stem loosening, 13 and 26 cases, respectively): comparing the results reported by 
Wacha et al. [20], we observed a higher rate of AL, but a lower rate of dislocations and PJI: a longer FU in our group of patients could be the explanation for a significant difference in cup survival due to AL. Figure 2 shows the radiographic 12 years $\mathrm{FU}$ in a patient with bilateral TOP cup paired with bilateral CFP stem.

In the majority of cases, the cup was paired with a CFP stem, in accordance with the principles of TSS: using a neck sparing stem such as the CFP allows the recreation of an artificial joint similar to the native hip, especially in terms of load transmission. This feature is important to prevent the stress shielding effect that could lead to implant mobilization [3].

Clinical evaluation showed excellent or good outcomes in the majority of patients (HHS, 86.3\% and OHS, 94,2\%), as previously described in shorter term FU studies [14, 21].

For every patient, an accurate preoperative planning was performed, in order to select the most suitable cup/stem
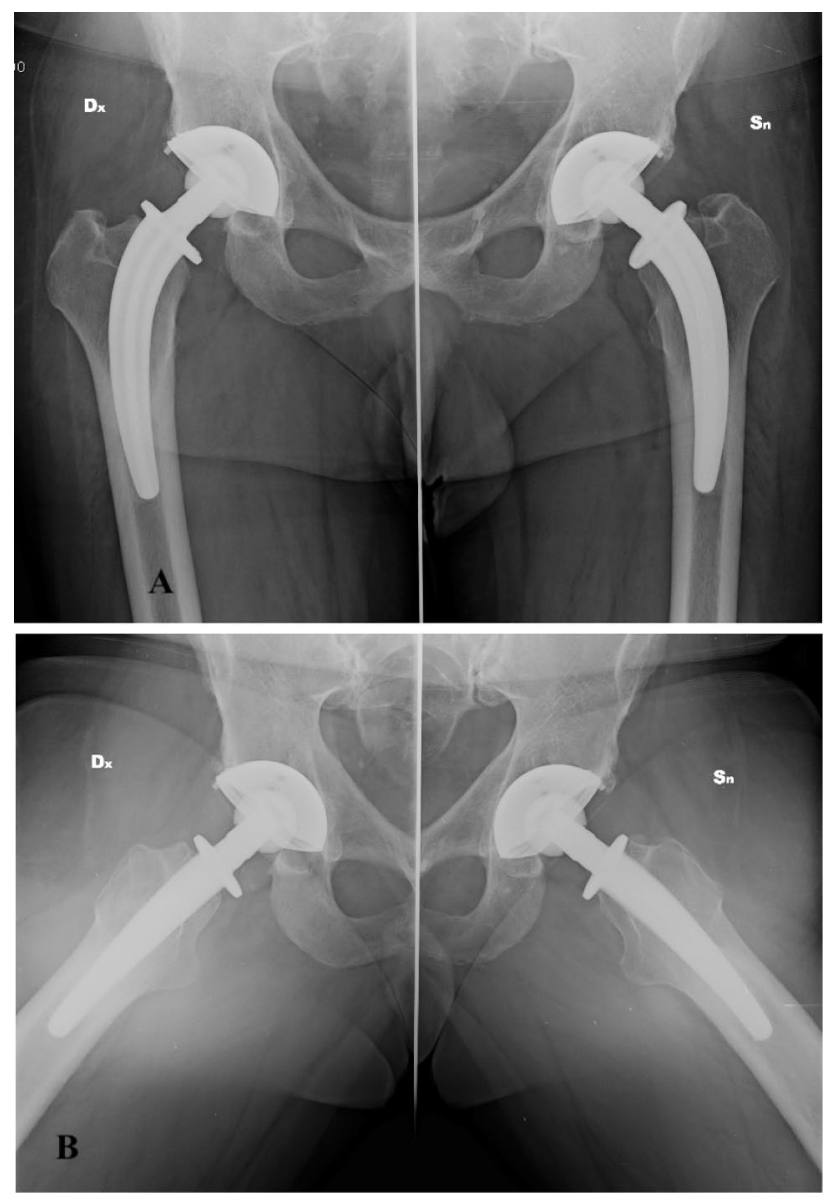

Fig. 212 years FU in a bilateral TOP cup, paired with CFP stem (Waldemar Link, Hamburg, Germany).a AP view. b Axial view of the stem. On the right side, a slight reabsorption of the femoral neck $(\mathrm{NRR}=0.18)$ pairing considering preoperative diagnosis, bone morphology and risk factors.

As previously indicated from Lazarinis et al. [22], TOP provides good stability, though proximal cancellous bone reabsorption is present in the early radiological evaluation after positioning. The significant correlation found between clinical and radiological outcomes, demonstrates how osteolysis in an active process in the operated hip and cannot be underestimated. The reduction in cancellous bone in Charnley and DeLee zones I and II is believed to be related to some sort of stress shielding: Gruen zones I and VII are the most affected on femoral side, accounting in combination for the $58.5 \%$ of all the osteolysis observed in the cohort, and it is current opinion that this pattern is referable to load transmission from the stem to the more distal part of the femur $[10,15,20,22,23]$. There were no records of psoas or femoral nerve impingement: the incidence of this complication using other cups is as high as $4.3 \%$ and $2.4 \%$, respectively $[24,25]$, and it is caused more often by the anteroinferior rim of the acetabulum. This complication could determine the necessity of a reoperation (e.g., cup reorientation or iliopsoas tendon tenotomy) [24]: the medio-ventral recess in the TOP cup avoids tendon and nerve irritation during hip flexion.

TOP cup permits an anatomical positioning even in complex acetabular, in which a more vertical implantation is required, achieving an adequate coverage thanks to its biequatorial feature [26, 27]. This is confirmed by the relatively low rate of dislocations $(0.7 \%)$ : in the literature, the incidence of dislocation following THA varies between $0.2 \%$ and $10 \%$ [28].

This study presents several limitations; first of all, its retrospective nature does not allow a control-case confrontation, in order to assess, for instance, differences in the use of other implants or different surgical techniques. Surgeries were performed by three different surgeons during a vast period of time; therefore, there could be biases on the final outcomes. Furthermore, the drop-out rate of $21,8 \%$ could not be ignored. Despite these limitations, the single-centre experience, long-term follow-up and the conspicuous number of patients are an undeniable strength: additionally, even though the procedures were performed by three different operators, surgical technique (i.e., surgical approach, component positioning) and pre- and postoperative care were the same for all the patients.

\section{Conclusions}

Uncemented press-fit cup is the preferred choice in contemporary hip replacement. Different designs are now available on the market. The TOP cup is a modern and low profile cup, which provides good clinical outcomes and reliability 
even for young patient over a long period of time. A precise preoperative planning and surgical technique are mandatory to achieve cup stability and lower revision rates. Further studies with similar FU on different cohorts of patients are necessary to confirm these results.

\section{Authors' contributions Not applicable.}

Funding Open access funding provided by Università degli Studi di Genova within the CRUI-CARE Agreement.

\section{Compliance with ethical standards}

Conflicts of interest The authors declare they have no relevant financial or non-financial interest to disclose.

Ethics approval All procedures performed in this study involving human participants were in accordance with the ethical standard of the institutional and/or national research committee and with the 1964 Declaration of Helsinki and its later amendments or comparable ethical standards.

Consent to participate Every patient gave his/her informed consent to participate to this retrospective study.

Consent to publication Every patient gave his/her informed consent to have his/her clinical records used for this retrospective study.

Summary of requirements The authors declare that they have no conflict of interest and no funding was received for this research article. The study is in accordance with institutional and national ethical standards, and all the participants gave their informed consent to their clinical information management.

Open Access This article is licensed under a Creative Commons Attribution 4.0 International License, which permits use, sharing, adaptation, distribution and reproduction in any medium or format, as long as you give appropriate credit to the original author(s) and the source, provide a link to the Creative Commons licence, and indicate if changes were made. The images or other third party material in this article are included in the article's Creative Commons licence, unless indicated otherwise in a credit line to the material. If material is not included in the article's Creative Commons licence and your intended use is not permitted by statutory regulation or exceeds the permitted use, you will need to obtain permission directly from the copyright holder. To view a copy of this licence, visit http://creativecommons.org/licenses/by/4.0/.

\section{References}

1. Learmonth ID, Young C, Rorabeck C (2007) The operation of the century: total hip replacement. Lancet 370:1508-1519. https://doi. org/10.1016/S0140-6736(07)60457-7

2. Villa T, Pipino F, Corradi A (2014) Tissue Sparing Surgery and Its Relevance within Hip Prosthesis. Open J Orthop 4:226-230

3. Pipino F, Calderale PM (1980) A biequatorial acetabular cup for hip prosthesis. Acta OrthopBelg 46:5-13
4. Pipino F, Cimmino M, Palermo A (2013) A modified direct lateral approach for neck-preserving total hip arthroplasty: tips and technical notes. J OrthopTraumatol 14(2):137-142

5. Nilsdotter A, Bremander A (2011) Measures of hip function and symptoms: Harris Hip Score (HHS), Hip Disability and Osteoarthritis Outcome Score (HOOS), Oxford Hip Score (OHS), Lequesne Index of Severity for Osteoarthritis of the Hip (LISOH), and American Academy of Orthopedic Surgeons (AAOS) Hip and Knee Questionnaire. Arthritis Care Res 63(Suppl 11):S200-S207. https://doi.org/10.1002/acr.20549

6. Chang CY, Huang AJ, Palmer WE (2015) Radiographic evaluation of hip implants. Semin Musculoskelet Radiol 19(1):12-20. https ://doi.org/10.1055/s-0034-1396763

7. DeLee JG, Charnley J.(1976) Radiological demarcation of cemented sockets in total hip replacement. ClinOrthopRelat Res (121):20-32

8. Gruen TA, McNeice GM, Amstutz HC (1979) Modes of failure of cemented stem-type femoral components: a radiographic analysis of loosening. ClinOrthopRelat Res 141:17

9. Brooker AF, Bowerman JW, Robinson RA, Riley LH Jr (1973) Ectopic ossification following total hip replacement. Incidence and a method of classification. J Bone Joint Surg Am 55:1629-1632

10. Formica M, Cavagnaro L, Basso M et al (2017) What is the fate of the neck after a collum femoris preserving prosthesis? A nineteen years single center experience. IntOrthop 41:1329-1335

11. Kjellberg M, Al-Amiry B, Englund E, Sjödén GO, Sayed-Noor AS (2012) Measurement of leg length discrepancy after total hip arthroplasty. The reliability of a plain radiographic method compared to CT-scanogram. SkeletalRadiol. 41(2):187-191. https:// doi.org/10.1007/s00256-011-1166-7

12. Paprosky WG, Perona PG, Lawrence JM (1994) Acetabular defect classification and surgical reconstruction in revision arthroplasty: a 6-year follow-up evaluation. J Arthroplasty 9:33-44. https://doi. org/10.1016/0883-5403(94)90135-X

13. Kress AM, Schmidt R, Nowak TE et al (2012) Stress-related femoral cortical and cancellous bone density loss after collum femoris preserving uncemented total hip arthroplasty: a prospective 7-year follow-up with quantitative computed tomography. ArchOrthop Trauma Surg 132(8):1111-1119. https://doi.org/10.1007/s0040 2-012-1537-0

14. Nowak M, Nowak TE, Schmidt R et al (2011) Prospective study of a cementless total hip arthroplasty with a collum femoris preserving stem and a trabeculae oriented press fit cup: minimum 6-year follow-up. Arch Orthop Trauma Surg 131:549-555

15. Briem D, Schneider M, Bogner N, Botha N, Gebauer M, Gehrke T, Schwantes B (2011) Mid-term results of 155 patients treated with a collum femoris preserving (CFP) short stem prosthesis. IntOrthop 35(5):655-660

16. Gill IR, Gill K, Jayasekera N, Miller J (2008) Medium term results of the collum femoris preserving hydroxyapatite coated total hip replacement [published correction appears in Hip Int. 2008 OctDec;18(4):335]. Hip Int. 18(2):75-80. https://doi.org/10.5301/ hip. 2008.2117

17. Schmidt R, Kress AM, Nowak M, Forst R, Nowak TE, Mueller LA (2012) Periacetabular cortical and cancellous bone mineral density loss after press-fit cup fixation: a prospective 7-year followup. J Arthroplasty 27(7):1358-1363.e1. https://doi.org/10.1016/j. arth.2011.09.031

18. Lazarinis S, Kärrholm J, Hailer NP (2010) Increased risk of revision of acetabular cups coated with hydroxyapatite: a Swedish Hip Arthroplasty Register study involving 8,043 total hip replacements. Acta Orthop 81:53-59

19. Lazarinis S, Mäkelä KT, Eskelinen A et al (2017) Does hydroxyapatite coating of uncemented cups improve long-term survival? An analysis of 28,605 primary total hip arthroplasty procedures from the Nordic Arthroplasty Register Association 
(NARA). Osteoarthritis Cartilage 25(12):1980-1987. https://doi. org/10.1016/j.joca.2017.08.001

20. Wacha H, Domsel G, Herrmann E (2018) Long-term follow-up of 1217 consecutive short-stem total hip arthroplasty (THA): a retrospective single-center experience. Eur J Trauma Emerg Surg 2018 44(3):457-469. https://doi.org/10.1007/s00068-017-0895-2

21. Kendoff D, Citak M, Egidy CC, O'Loughlin PF, Gehrke T (2013) Eleven-year results of the anatomic coated CFP stem in primary total hip arthroplasty. J Arthroplasty 28(6):1047-1051

22. Lazarinis S, Milbrink J, Mattsson P, Mallmin H, Hailer NP (2014) Bone loss around a stable, partly threaded hydroxyapatite-coated cup: a prospective cohort study using RSA and DXA. Hip Int 24(2):155-166

23. Gillies RM, Kohan L, Cordingley R (2007) Periprosthetic bone remodelling of a collum femoris preserving cementless titanium femoral hip replacement. Comput Methods Biomech Biomed Eng 10(2):97-10

24. Dora C, Houweling M, Koch P, Sierra RJ (2007) Iliopsoas impingement after total hip replacement: the results of non-operative management, tenotomy or acetabular revision. J Bone Joint SurgBr 89(8):1031-1035. https://doi. org/10.1302/0301-620X.89B8.19208
25. Fox AJ, Bedi A, Wanivenhaus F, Sculco TP, Fox JS (2012) Femoral neuropathy following total hip arthroplasty: review and management guidelines. Acta OrthopBelg 78(2):145-151

26. Pipino F (2004) CFP prosthetic stem in mini-invasive total hip arthroplasty. J OrthopaedTraumatol 5:165-171. https://doi. org/10.1007/s10195-004-0065-2

27. van Oldenrijk J, Schafroth MU, Bhandari M et al (2008) Timeaction analysis (TAA) of the surgical technique implanting the collum femoris preserving (CFP) hip arthroplasty. TAASTIC trial identifying pitfalls during the learning curve of surgeons participating in a subsequent randomized controlled trial (an observational study). BMC Musculoskelet Disord. 9:93. https:// doi.org/10.1186/1471-2474-9-93

28. Dargel J, Oppermann J, Brüggemann GP, Eysel P (2014) Dislocation following total hip replacement. DtschArzteblInt 111:884-890

Publisher's Note Springer Nature remains neutral with regard to jurisdictional claims in published maps and institutional affiliations. 